\title{
Los géneros Docidium, Penium, Haplotaenium, Pleurotaenium, Tetmemorus y Triploceras (Desmidiales, Chlorophyta) en Cuba
}

\author{
Víctor M. Martínez Almeida
}

Centro de Investigaciones Algológicas de Cienfuegos (CITMA). Cienfuegos, Cuba e-mail: vmma@labcost.perla.inf.cu

\begin{abstract}
Resumen. Este trabajo es una continuación de los estudios sobre las desmidiáceas de Cuba. Fueron examinadas 160 muestras recolectadas entre los años 1979 y 1988 de las zonas más importantes desde el punto de vista de presencia de desmidiáceas y diversidad de microalgas en Cuba, viz.: Pinar del Río, Isla de la Juventud, Ciénaga de Zapata, Matanzas y San Felipe, Camagüey. Con especial interés se profundizó en las de forma cilíndrica, determinando 22 taxa infragenéricos pertenecientes a 17 especies, de los cuales 18 son nuevos registros para Cuba. Algunas poblaciones mostraron amplia variabilidad morfométrica. Se describe brevemente cada taxón, con ilustraciones originales, algunos comentarios sobre su distribución y sinonimia más importante.
\end{abstract}

Palabras claves: Desmidiales, desmidiáceas, géneros cilíndricos, Cuba.

\begin{abstract}
This is a further contribution to the knowledge of the desmid flora of Cuba. One hundred and sixty samples collected from the most important regions in regard to occurrence of desmids and diversity of microalgae species in Cuba (Pinar del Río, Isla de la Juventud, Ciénaga de Zapata, Matanzas and San Felipe, Camagüey), were examined. Emphasis was made on the taxonomic survey of those "cylindrical" representative genera. 22 infrageneric taxa belonging to 17 species were identified, 18 of which are new records from the country. Some populations examined showed a wide morphological variability. Each taxon is briefly described based on Cuban material. Original drawings and comments about distribution and synonymy according to the available taxonomic literature are included.
\end{abstract}

Key words: Desmidiales, desmids, cylindrical genera, Cuba.

L a flora desinidiológica, así como la de otros grupos de algas dulceacuícolas es poco conocida en Cuba, por ello existe la necesidad de estudios taxonómicos fundamentales para entender mejor temas tan importantes como la biodiversidad y la ecología. En términos generales, las desmidiáceas presentan una distribución amplia; pero se ha comprobado que muchas de ellas sólo se encuentran en determinados ambientes, y presentan variaciones morfológicas respecto a las características taxonómicas importantes. Este grupo constituye, a su vez, por su alta sensibili- dad ante diferentes variables físicas y químicas, excelentes bioindicadores de la calidad de los medios acuáticos.

En Cuba alcanzan una gran diversidad, principalmente en aguas de bajo contenido mineral, características de algunas localidades de Pinar del Río, Isla de la Juventud, Ciénaga de Zapata, Matanzas y San Felipe, Camagüey.

Como parte de la continuación de los estudios florísticos y taxonómicos sobre las desmidiáceas de Cuba (Martínez, 1989 a,b,c) se registran en este trabajo los 


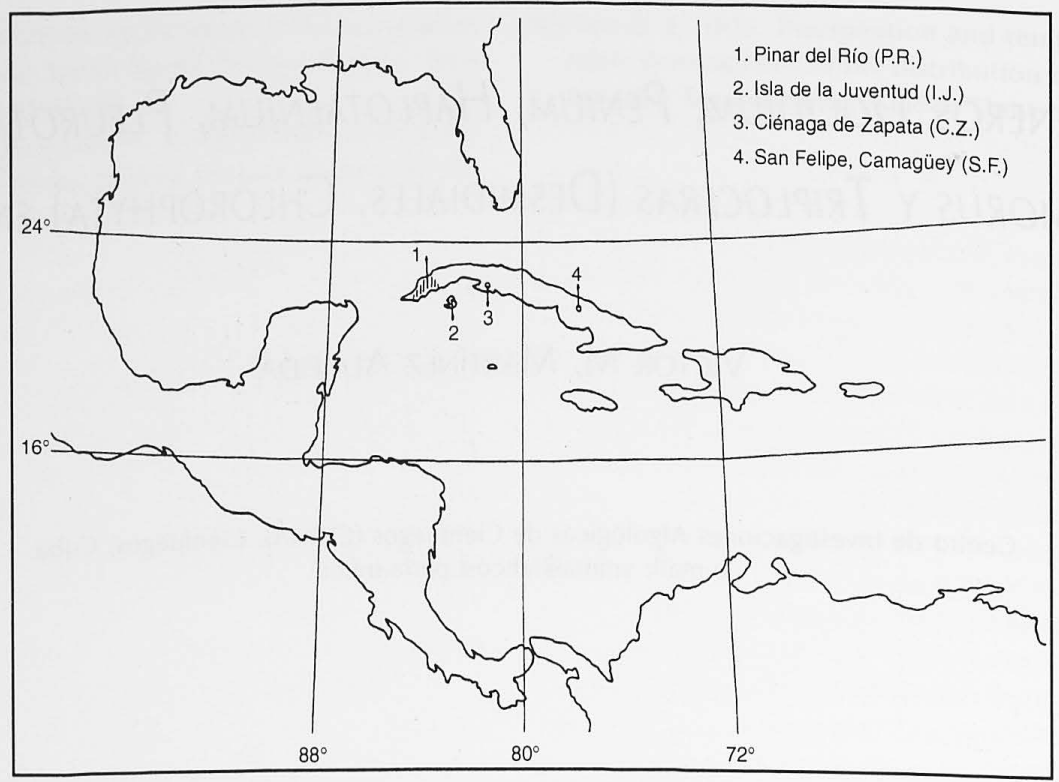

Figura 1. Regiones mejor representadas por desmidiáceas en Cuba.

taxa de los géneros de forma cilíndrica: Docidium Brébisson, Penium Brébisson, Haplotaenium Bando, Pleurotaenium Nägeli, Tetmemorus Ralfs y Triploceras Bailey.

\section{Materiales y métodos}

Se revisaron 160 muestras recolectadas de los años 1979 a 1988 en diferentes localidades y épocas del año de las cuatro regiones en donde están mejor representadas las desmidiáceas en Cuba: Pinar del Río (P.R.) e Isla de la Juventud (I.J.), zona occidental del país; Ciénaga de Zapata, Matanzas (C.Z.), zona centro - sur (cenagosa) y San Felipe, Camagüey (S.F.), región centro oriental de la Isla (figura 1).

En el análisis se incluyen muestras del plancton (obtenido con una malla de $10 \mu \mathrm{m}$ ), del perifiton obtenido al exprimir plantas acuáticas litorales (especies de Utricularia, Potamogeton, Ceratophyllum, Eichhornia y otras) o del detrito del fondo de acuatorios con poca profundidad.

El material preservado con formaldehido al $4 \%$ permanece en la colección del Laboratorio de Investigaciones Algológicas de Cienfuegos, perteneciente al Ministerio de la Ciencia, Tecnología y Medio Ambiente, Cuba.

Las ilustraciones de los organismos encontrados se realizaron en un microscopio Amplival y una cámara clara Carl Zeiss - Jena.
En el presente trabajo hemos seguido al sistema taxonómico propuesto por Round (1971).

\section{Resultados y discusión}

Docidium Brébisson ex Ralfs, 1848, p. 155, pro parte, emend. Lundell, 1871, p. 88.

D. baculum Brébisson ex Ralfs, 1848 , p. 158, pl. 33, figura 5. (figura 2, a - d).

Células 18.5-26.5 veces más largas que anchas, 185$316 \times 9.5-12 \mu \mathrm{m}$, istmo $7-9 \mu \mathrm{m}$. Material examinado: P.R. (438, 445-B); I.J. (81-52, 84-3A); S.F. (34B-88; 53B-88, 80A-88, 44A-89). Hábitat: perifiton.

Penium Brébisson ex Ralfs, 1848, p.148 emend. Kouwets et Coesel, 1984, p.561.

P margaritaceum (Ehrenberg) Brébison ex Ralfs, 1848, p. 149, pl. 25, figura 1a-c, e-h , pl. 33, figura 3. (figura $2, \mathrm{e})$.

Basónimo: Closterium margaritaceum Ehrenberg, 1838, p. 95, pl. 6, figura 13.

Células 4-6 veces más largas que anchas, $66-111 \mathrm{X}$ 17-19 $\mu \mathrm{m}$, ancho del ápice $11-15 \mu \mathrm{m}$, istmo $16-18 \mu \mathrm{m}$. Material examinado: P.R. (458). Hábitat: perifiton.

P. spirostriolatum Barker, 1869, p. 194. (figura 2, f - g). Células 5.5-10 veces más largas que anchas, 100-155 X 13-19 $\mu \mathrm{m}$, ápice 10-16 $\mu \mathrm{m}$ de ancho, istmo 10- 


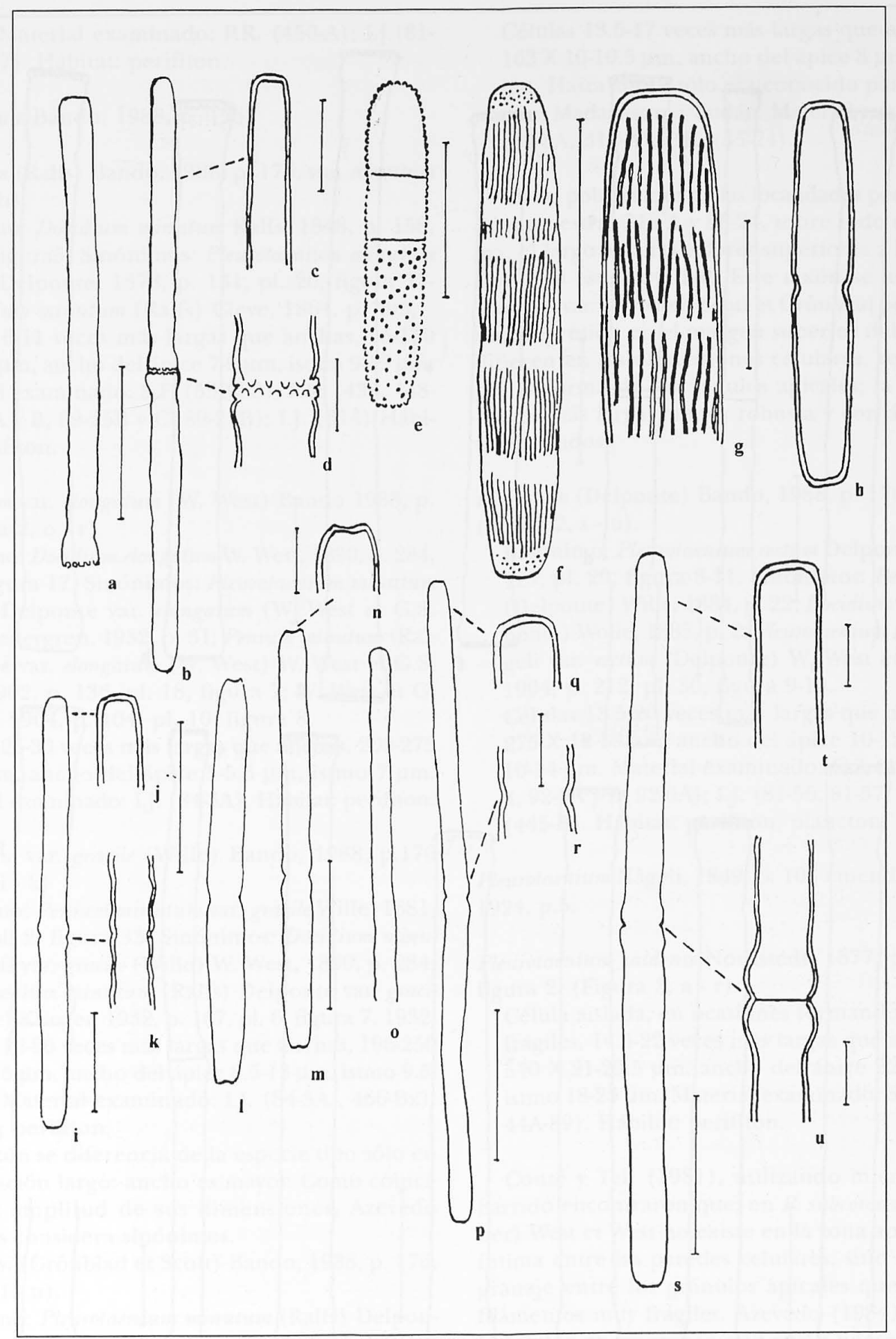

Figura 2. a - d: Docidium baculum var. baculum; c: detalle del ápice; d: detalle del istmo; e: Penium margaritaceum; f g: P. spirostriolatum; h: Haplotaenium minutum var. minutum; i - k: H. minutum var. gracile; j: detalle del ápice; k: detalle del istmo; $1-\mathrm{n}:$ H. bourrellyi; $\mathrm{n}$ : detalle del ápice; o - r: H. minutum var. elongatum; q: detalle del ápice; r: detalle del istmo; s - u: $H$. rectum var. rectum; t: detalle del ápice; $\mathrm{u}$ : detalle del istmo; Escalas: d, n, q, r, t, u: barra = 10 $\mu \mathrm{m}$. h: barra $=20 \mu \mathrm{m}$, otras: barra $=50 \mu \mathrm{m}$. 
Victor M. Martinez Almeida

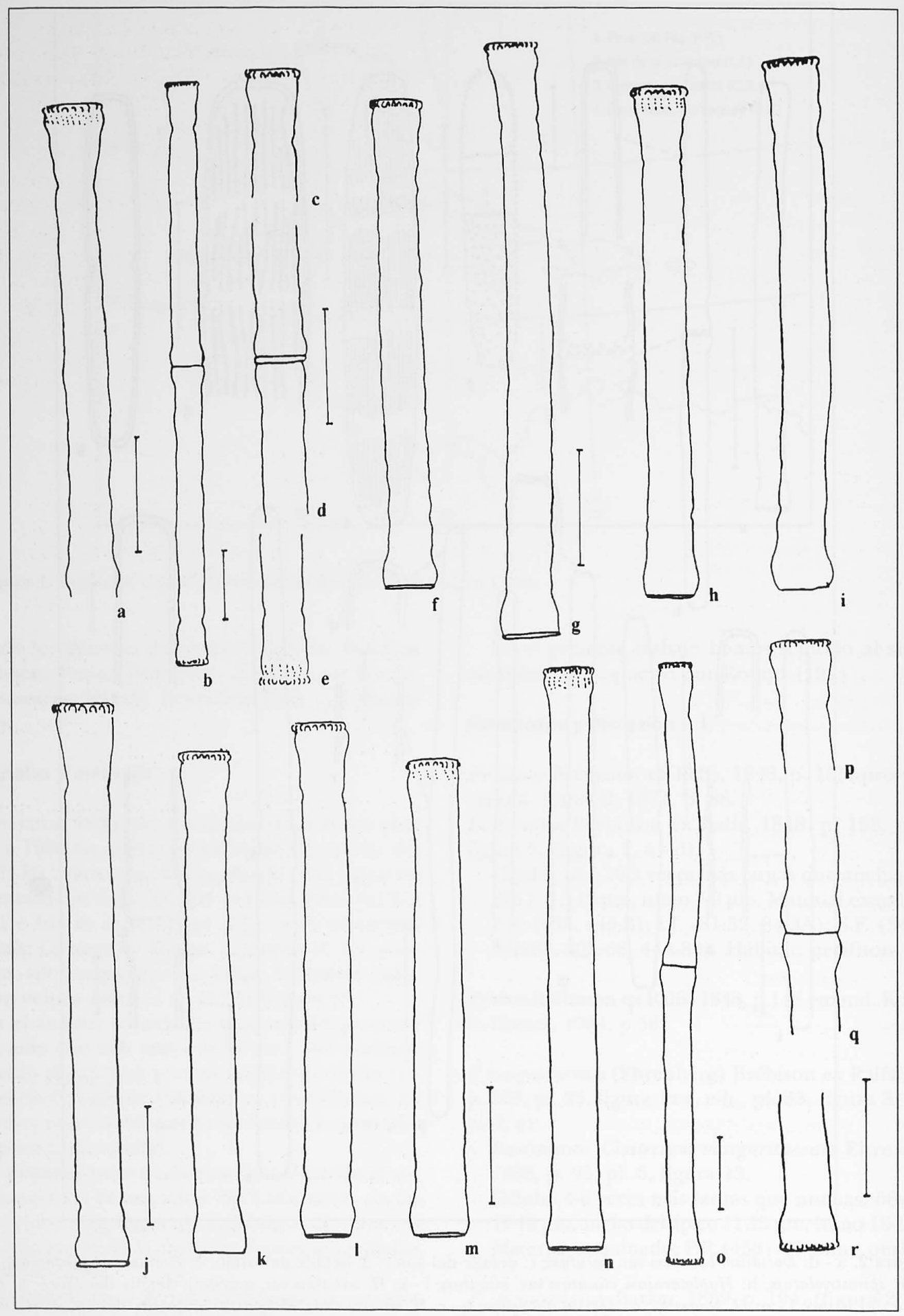

Figura 3. a - r: Pleurotaenium caldense; c, e, p, r: detalles del ápice; d, q: detalles del istmo. Escala: barra $=50 \mu \mathrm{m}$. 
$15 \mu \mathrm{m}$. Material examinado: P.R. (450-A); I.J. (8156, 81-57). Hábitat: perifiton.

Haplotaenium Bando, 1988, p. 176.

H. minutum (Ralfs) Bando, 1988, p. 176, var. minutum (figura 2, h).

Basónimo: Docidium minutum Ralfs, 1848, p. 158, pl. 26, figura5. Sinónimos: Pleurotaenium minutum (Ralfs) Delponte, 1878 , p. 131, pl. 20, figura 1721; Penium minutum (Ralfs) Cleve, 1864, p. 493. Células 6-11 veces más largas que anchas, 65-150 $\times 10-14 \mu \mathrm{m}$, ancho del ápice 7-9 $\mu \mathrm{m}$, istmo 9-12 $\mu \mathrm{m}$. Material examinado: S.F. (53B-88); P.R. (438, 448B, 87-8A y B, 89-25B y C, 89-27B); I.J. (314) Hábitat: perifiton.

H. minutum var. elongatum (W. West) Bando 1988, p. 176 (figura 2, o - r).

Basónimo: Docidium elongatum W. West, 1890, p. 284, pl. 5, figura 17. Sinónimos: Pleurotaenium minutum (Ralfs) Delponte var. elongatum (W. West et G.S. West) Cedergren, 1932, p. 31; Penium minutum (Ralfs) Cleve var. elongatum (W. West) W. West et G.S. West, 1902, p. 136, pl. 18, figura 7; W. West et G. S. West, 1904, p. 104, pl. 10, figura 8.

Células 25-30 veces más largas que anchas, 200-273 $X$ 8-9 $\mu \mathrm{m}$, ancho del ápice $5-5.5 \mu \mathrm{m}$, istmo $7 \mu \mathrm{m}$. Material examinado: I.J. (84-3A). Hábitat: perifiton.

H. minutum var. gracile (Wille) Bando, 1988, p.176 (figura 2, i - k)

Basónimo: Penium minutum var. gracile Wille, 1881, p. 51, pl. 2, figura 33. Sinónimos: Docidium minutum Ralfs var. gracile (Wille) W. West, 1890, p. 284; Pleurotaenium minutum (Ralfs) Delponte var. gracile (Wille) Krieger, 1932, p. 167, pl. 6, figura 7. 1932. Células 16-20 veces más largas que anchas, 196-250 $\times 10.5-15 \mu \mathrm{m}$, ancho del ápice $8.5-13 \mu \mathrm{m}$, istmo $9.5-$ $13 \mu \mathrm{m}$. Material examinado: I.J. (84-3A , 466-Bx). Hábitat: perifiton.

Este taxón se diferencia de la especie tipo sólo en que la relación largo: ancho es mayor. Como coinciden en la amplitud de sus dimensiones, Azevedo (1984), las considera sinónimos.

H. bourrellyi (Grönblad et Scott) Bando, 1988, p. 176, (figura 2, $1-n$ ).

Basónimo: Pleurotaenium minutum (Ralfs) Delponte var. bourrellyi Grönblad et Scott in Grönblad, Prowse et Scott, 1958, p. 11, pl. 1, figura 2-3; pl. 24, foto 341. 1958. Sinónimos: Pleurotaenium minutum (Ralfs) Delponte var. minus Bourrelly, in Bourrelly et Leboime, 1946, p. 83, pl. 2, figura 18. (no var. minus (Raciborski) Krieger, 1932, p.168).
Células 13.5-17 veces más largas que anchas, 136$163 \times 10-10.5 \mu \mathrm{m}$, ancho del ápice $8 \mu \mathrm{m}$, istmo 9.5 $\mu \mathrm{m}$. Hasta ahora sólo era conocido para Brasil, Japón, Madagascar, y Sudán. Material examinado: I.J. (84-8A, 312-A); P.R. (85-24).

En las poblaciones de las localidades pertenecientes a las muestras 312-A y 85-24, sobre todo en ésta última, el largo alcanza valores superiores a los registrados para esta variedad. Este taxón se asemeja a $P$. minutum var. excavatum Scott et Grönblad por presentar una depresión en el margen superior del ápice pero difieren en las dimensiones celulares, relación l:a y por la forma de los ángulos apicales; la var. excavatum es más larga, menos robusta y con ángulos apicales agudos.

H. rectum (Delponte) Bando, 1988, p. 176 var. rectum (figura 2, s-u).

Basónimo: Pleurotaenium rectum Delponte, 1878, p. 129, pl. 20, figura 8-11. Sinónimos: Penium rectum (Delponte) Wille, 1884, p. 22; Docidium rectum (Delponte) Wolle, 1885 , p. 2 ; Pleurotaenium trabecula Nägeli var. rectum (Delponte) W. West et G.S. West, 1904, p. 212, pl. 30, figura 9-10.

Células 13.5-20 veces más largas que anchas, 176$275 \times 12-16 \mu \mathrm{m}$, ancho del ápice $10-12 \mu \mathrm{m}$, istmo 10-14 $\mu \mathrm{m}$. Material examinado: C.Z.(487, 492, 924, 92-5A y B, 92-6A); I.J. (81-56, 81-57, 81-40); P.R. (445-B). Hábitat: perifiton, plancton.

Pleurotaenium Nägeli, 1849, p. 104 emend. Grönblad, 1924 , p.5.

Pleurotaenium caldense Nordstedt, 1877, p. 17, pl. 2, figura 2. (Figura 3, a - r).

Célula aislada, en ocasiones formando filamentos frágiles, 14.5-22 veces más largas que anchas, 380$540 \times 21-29.5 \mu \mathrm{m}$, ancho del ápice 22.5-29.5 $\mu \mathrm{m}$, istmo $18-23 \mu \mathrm{m}$. Material examinado: S.F. (53B-88, 44A-89). Hábitat: perifiton.

Couté y Tell (1981), utilizando microscopía de barrido encontraron que, en $P$. subcoronulatum (Turner) West et West no existe en la zona apical ligazón íntima entre las paredes celulares, sino sólo un engranaje entre los gránulos apicales que hace a los filamentos muy frágiles. Azevedo (1984), cuestionó la validez de mantener este taxón como especie independiente de $P$. caldense sólo por ser la primera de hábito filamentoso, ambas coinciden en cuanto a su morfología y morfometría. West y West (1896), describieron $P$. subcoronulatum var. detum que se caracteriza por la presencia de una fuerte constricción en 


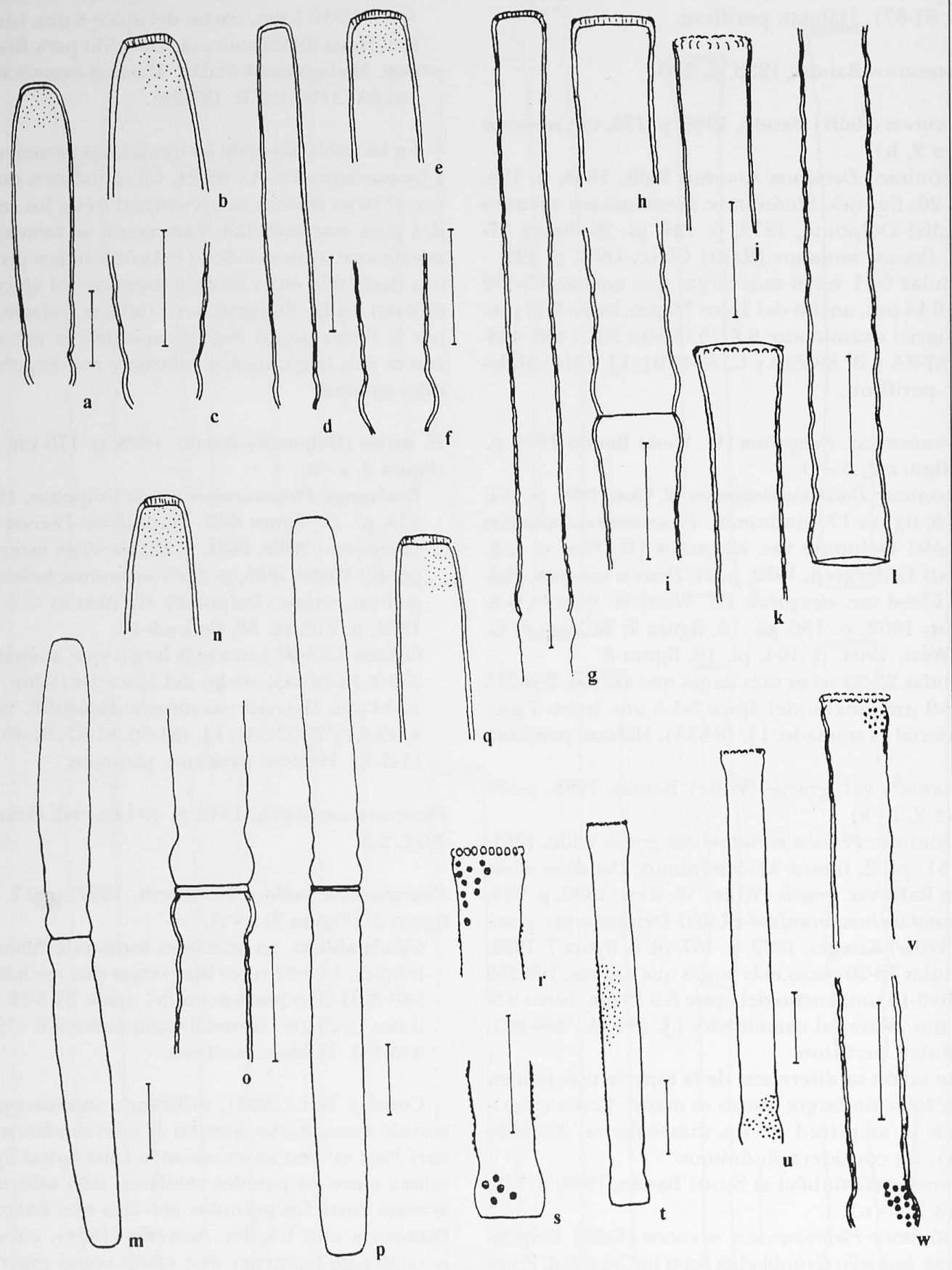

Figura 4. a - f, m - q: Pleurotaenium trabecula; b, e, n, q: detalles del ápice; c, f, o: detalles del istmo; g - 1: $P$. simplicissimum var. insigne; $\mathrm{h}, \mathrm{j}$, k: detalles del ápice; i: detalle del istmo; l: detalle de una hemicélula; $\mathrm{r}-\mathrm{w}$ : $P$. caldense var. granulatum; r, v: detalles del ápice; s, w: detalles del istmo. Escala: barra $=50 \mu \mathrm{m}$. 
la región subapical de las hemicélulas y por los márgenes laterales ondulados por encima de la intumescencia basal. Borge (1903), observó en un taxón, que él describió como P. subcorolunatum var. rectum, la presencia de una constricción suave en loṣ márgenes laterales de algunos individuos, no tan acentuada como en la var. detum. Azevedo (1984), consideró a la var. rectum como sinónimo de $P$. caldense var. cristatum. Para el Estado de Pará, Brasil, Grönblad (1945), observó que en las hemicélulas de $P$. caldense Nordstedt aparecen los márgenes laterales con ondulaciones muy suaves e irregulares y una leve constricción por debajo del ápice bulboso, carácter que nunca había sido mencionado en la literatura para tal especie. Desafortunadamente, Grönblad (1945) no presentó ilustración.

En muestras recolectadas en el Estado de São Paulo, Brasil, Azevedo (1984), encontró poblaciones de $P$. caldense var. cristatum en las que aparecieron individuos con márgenes apicales divergentes y subapicales con una constricción extremadamente suave o más acentuada (Azevedo, 1984; figuras 114a, 115 y. 111, respectivamente).

En Cuba los especímenes de dos poblaciones de una misma localidad (San Felipe, Camagüey), que rara vez forman filamentos de dos células, presentaron inestabilidad con respecto a la constricción subapi$\mathrm{cal}$, que iba desde su total presencia (figura $3, \mathrm{a}-\mathrm{g}$ ), presente sólo en alguna de las zonas subapicales (figura 3, o) o totalmente ausentes (figura $3, \mathrm{~m}, \mathrm{n}$ ). Igual a lo observado por otros autores, esta constricción, no era tan acentuada como la registrada para $P$. subcoronulatum var. detum. La morfometría, sobre todo el ancho máximo de la hemicélula, está por debajo, o en los límites inferiores a los registrados en la literatura. De igual forma, el $26.7 \%$ de los individuos no presentaron ondulación alguna sobre la intumescencia basal. Otros, incluso presentaron una ligera constricción sobre ella (figura $3, \mathrm{~h}-1$ ). Por poseer esta amplia variabilidad morfológica, este taxón es de difícil ubicación Coincidimos con los resultados de Azevedo (1984), de no considerar convincente la separación de $P$. caldense de $P$. subcoronulatum. Para esclarecer tal separación, teniendo en cuenta la amplia variabilidad morfológica, se requiere de más estudios donde pudieran también determinarse las posibles condiciones ecológicas que influyan en estos caracteres tan variables.

P. caldense Nordstedt var. granulatum Borge, 1903, p. 82, pl. 2, figura 4. (figura $4, \mathrm{r}-\mathrm{w}$ ).

Células 16.5-22 veces más largas que anchas, 484$651 \times 29-31 \mu \mathrm{m}$, ancho del ápice $30-31 \mu \mathrm{m}$, istmo 26.5-28 $\mu \mathrm{m}$. Material examinado: I.J. (466-Bx).. Hábitat: perifiton.
La var. granulatum descrita por Borge (1903), fue considerada por Krieger (1937), dentro de la variedad tipo de la especie. Posteriormente, Grönblad (1945), la registró de nuevo como $P$. caldense var. granulatum para el estado de Pará, Brasil. Azevedo (1984), consideró que su ilustración no aclara bien la identidad de este taxón y lo siguió tratando como sinónimo de $P$. caldense var. caldense.

La presencia de gránulos en las paredes celulares, lo que la diferencia de la variedad tipo, aparece de manera estable en los individuos de la población cubana estudiada, por lo que consideramos se trata realmente de un taxón independiente que se conocía anteriormente sólo para el Paraguay y Brasil.

P. constrictum (Bailey) Wood, 1873, p. 121. (figura 5, $\mathrm{u}-\mathrm{w})$.

Basónimo: Docidium constrictum Bailey in Ralfs, 1848, p.218, pl. 35, figura 7 .

Células 8-9 veces más largas que anchas, 417-445 X 50-52 $\mu \mathrm{m}$, ancho del ápice 31-32 $\mu \mathrm{m}$, istmo 36$38 \mu \mathrm{m}$. Material examinado: I.J. (84-12). Hábitat: perifiton. Hasta ahora sólo estaba registrada para Norteamérica.

P. ehrenbergii (Brébisson ex Ralfs) De Bary, 1858, p.75 var. ehrenbergii (figura 5, a - f).

Basónimo: Docidium ehrenbergii Brébisson ex Ralfs, 1848 , p. 157, pl. 26 , figura 4.

Células 12-14 veces más largas que anchas, 410-444 $\times 31-33 \mu \mathrm{m}$, ancho del ápice $20-22 \mu \mathrm{m}$, istmo $26.5-$ $28 \mu \mathrm{m}$. Material examinado: P.R. (445-B, 460, 874, 87-6, 87-8A, 87-8B, 89-26A y B, 89-27B); I.J.(404). Hábitat: perifiton.

P. ehrenbergii (Brébisson ex Ralfs) De Bary var. elongatum (W. West) W. West, 1892, p. 119. (figura 5, g - l).

Basónimo: Docidium ehrenbergii Brébisson var. elongatum W. West, 1890, p. 284.

Células 20-29 veces más largas que anchas, (325)364-540 X 15-22 $\mu \mathrm{m}$, ancho del ápice 11-15 $\mu \mathrm{m}$, istmo 10-18 $\mu \mathrm{m}$. Material examinado: S.F. (53B-88, 78C-88); I.J. (81-57); P.R. (445-B, 458, 85-22, 89-25B, 89-28B). Hábitat: perifiton.

El ancho máximo de la hemicélula, así como el ancho del ápice se encuentran por debajo o en el límite inferior de lo registrado en la literatura. No obstante, se considera dentro de la var. elongatum por encontrarse dentro de la amplitud de la relación largo: ancho descrito para esta variedad.

P. ehrenbergii (Brébisson) De Bary var. undulatum Schaarschmidt, 1883, p. 278, pl. 1, figura 21. (figura $5, \mathrm{~m}-\mathrm{p})$. 


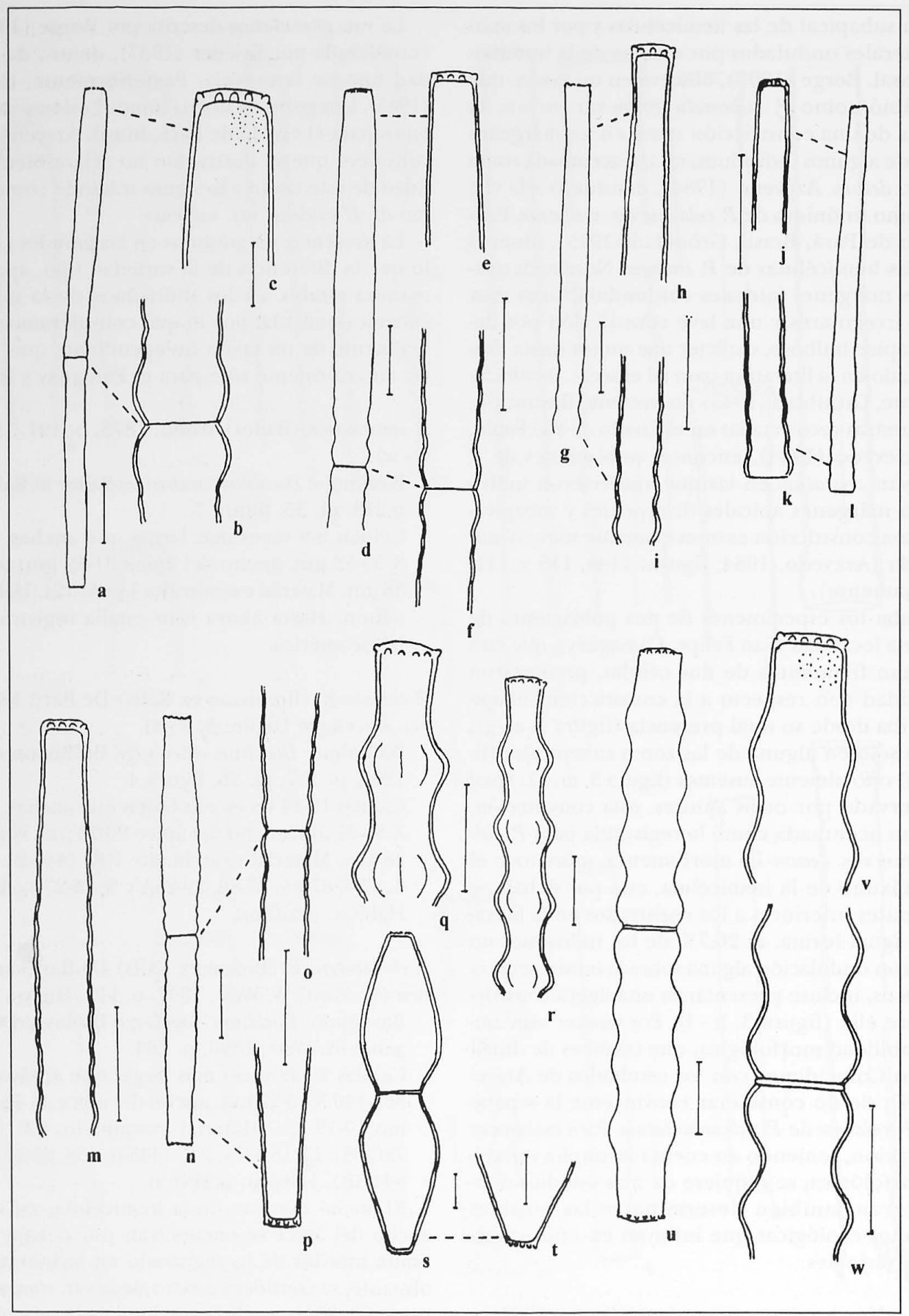

Figura 5. a - f: Pleurotaenium ehrenbergii var. ehrenbergii; c, e: detalles del ápice; b, f: detalles del istmo; g - l: $P$. ehrenbergii var. elongatum; $\mathrm{h}, \mathrm{j}$ : detalles del ápice; $\mathrm{i}, \mathrm{k}$ : detalles del istmo; $\mathrm{m}-\mathrm{p}$ : P. elirenbergii var. undulatum; m: detalle de una hemicélula; o: detalle del istmo; $\mathrm{p}$ : detalle del ápice; q -r: $P$. nodosum var. borgei; q: detalle del ápice; $\mathrm{s}$-t: $P$. ovatum; t: detalle del ápice; $\mathrm{u}-\mathrm{w}$ : $P$. constrictum; $\mathrm{v}$ : detalle del ápice; w: detalle del istmo. Escalas: b, $\mathrm{c}:$ barra $=20$ $\mu \mathrm{m}$. Otras: barra $=50 \mu \mathrm{m}$. 
Células (8)-11-17 veces más largas que anchas, 223$434 \times 26-30 \mu \mathrm{m}$, ancho del ápice $18.5-20 \mu \mathrm{m}$, istmo 20-23 $\mu \mathrm{m}$. Material examinado: P.R. (460). Hábitat: perifiton.

P. elatum (Turner) Borge var. alternans (Nordstedt) Grönblad, 1945, p. 11, pl. 2, figura 25. (figura 6, a -b). Basónimo: Docidium alternans Nordstedt, 1870, p. 205, pl. 3, figura 36 .

Células 14-14.5 veces más largas que anchas, 700$730 \times 49-52 \mu \mathrm{m}$, ancho del ápice $48-51 \mu \mathrm{m}$, istmo 41-42.5 $\mu \mathrm{m}$. Material examinado: I.J. (306-B). Hábitat: perifiton.

Hasta ahora sólo era conocida para Brasil.

P. nodosum (Bailey ex Ralfs) Lundell var. borgei (Grönblad)

Krieger, 1937, p. 437, pl. 47, figura 4. (figura 5, q-r).

Basónimo: Pleurotaenium nodosum f. borgei Grönblad, 1920, p. 27, pl. 4, figura 28. 1920.

Células 9-9.5 veces más largas que anchas, 450-480 $X$ 48-52 $\mu \mathrm{m}$, ancho del ápice $33-34 \mu \mathrm{m}$, istmo 26.5 $28 \mu \mathrm{m}$. Material examinado: I.J. (403-B, 81-52). Hábitat: perifiton.

P. ovatum (Nordstedt) Nordstedt, 1877 , p. 18 (figura $5, \mathrm{~s}-\mathrm{t})$.

Basónimo: Docidium ovatum Nordstedt, 1870, p. 205, pl.3, figura $37 \mathrm{a}$

Células 3-3.5 veces más largas que anchas, 388-437 $X 123-127 \mu \mathrm{m}$, ancho del ápice $31-32 \mu \mathrm{m}$, istmo 65 $69 \mu \mathrm{m}$. Material examinado: P.R. (87-4, 87-6B, 8525B). Hábitat: perifiton.

Sólo conocido para regiones tropicales y subtropicales.

P. simplicissimum Grönblad var. insigne (Roll) Krieger, 1937, p. 419, pl. 44, figura 10. 1937. (figura 4, g - l). Basónimo: Pleurotaenium insigne Roll, 1923, p. 41, figura 12

Células 22-27 veces más largas que anchas, $880-1000$ $\times 36-40 \mu \mathrm{m}$, ancho del ápice $32-39 \mu \mathrm{m}$, istmo 31 $35 \mu \mathrm{m}$. Material examinado: P.R. (448-B). Hábitat: plancton.

P. simplicissimum var. insigne se parece mucho a $P$. gloriosum en cuanto a dimensiones, hábito y número de gránulos en el ápice, mas difiere de éste por la intumescencia basal no conspicua y por los márgenes laterales irregulares en toda su extensión. Hasta el momento sćlo registrada para Europa y Brasil.

Pleurotaenium trabecula (Ehrenberg) ex Nägeli, 1849, p. 104. (figura 4, a - f, m- q).

Basónimo: Closterium trabecula Ehrenberg, 1830, p. 62, 70. Sinónimo: C. trabecula Ehrenberg, 1838, p. 93 pro parte, pl. 6:II, 7 .
Células 12-17.5 veces más largas que anchas, 395$690 \times 31-40 \mu \mathrm{m}$, ancho del ápice $20-30 \mu \mathrm{m}$, istmo 25-33 $\mu \mathrm{m}$. Material examinado: P.R. (445-A,B; 874); C.Z. (496, 484-A, 91-30, 91-41, 92-5A); I.J. (8152, 84-12). Hábitat: perifiton.

Existe una gran variabilidad morfológica en las diferentes poblaciones estudiadas.

P. verrucosum (Bailey ex Ralfs) Lundell, 1871, p. 6 (figura $6, \mathrm{f}-\mathrm{h}$ ).

Basónimo: Docidium verrucosum (Bailey) ex Ralfs, 1848, p. 218. Sinónimo: Closterium verrucosum Bailey, 1846 , p. 127, figura 4.]

Células $10-15$ veces más largas que anchas, 310-360 $X$ 24-33 $\mu \mathrm{m}$, ancho del ápice 18-30 $\mu \mathrm{m}$, istmo 20$26 \mu \mathrm{m}$. Material examinado: P.R. (448-B, 438); I.J. (403, 81-40, 81-52, 81-56, 84-3A). Hábitat: perifiton.

Tetmemorus Ralfs, 1848, p. 145.

T. granulatus (Brébisson) Ralfs, 1848, p. 147, pl. 24, figura 2; pl. 33, figura 1. (figura 6 , i - k)

Basónimo: Closterium granulatum Brébisson, 1839, p. 272]

Células fusiformes en vista lateral y frontal, $4-5$ veces más largas que anchas, 92-105 X 23-25 $\mu \mathrm{m}$, ancho del ápice 11-12 $\mu \mathrm{m}$, istmo $21-23.5 \mu \mathrm{m}$, grosor 24 $\mu \mathrm{m}$. Material examinado: I.J. (81-57, 314). Hábitat: perifiton.

Triploceras Bailey, 1851, p. 37. 1851.

T. verticillatum Bailey, 1851, p. 37, pl. 1, figura 9. (figura $6, c-e)$.

Células $10-20$ veces más largas que anchas, 356-615 X 31-42 $\mu \mathrm{m}$, ancho del ápice más espinas $63.5-66$ $\mu \mathrm{m}$, istmo $20-28 \mu \mathrm{m}$. Material examinado: I.J. (466Bx, 84-3A); P.R. (438, 445-B, 448-C, 87-8B). Hábitat: perifiton.

Especie conocida para América y Gran Bretaña. Anteriormente registrada para Cuba por Lagerheim (1885).

Entre los 22 taxa señalados en el presente trabajo el $50 \%$ son de posible distribución amplia. Sin embargo, según la literatura disponible, Haplotaenium bourrelly, Pleurotaenium caldense var. granulatum, $P$. constrictum var. constrictum, $P$. elatum var. alternans, $P$. simplicissimum var. insigne y Triploceras verticillatum están circunscritos a zonas bien delimitadas del mundo. Según los resultados actuales, los taxa de hábito cilíndrico más frecuentemente observados en Cuba son: $H$. rectum var. rectum, $P$. ehrenbergii var. ehrenbergii y $P$. trabecula var. trabecula. Algunos como P. caldense, 


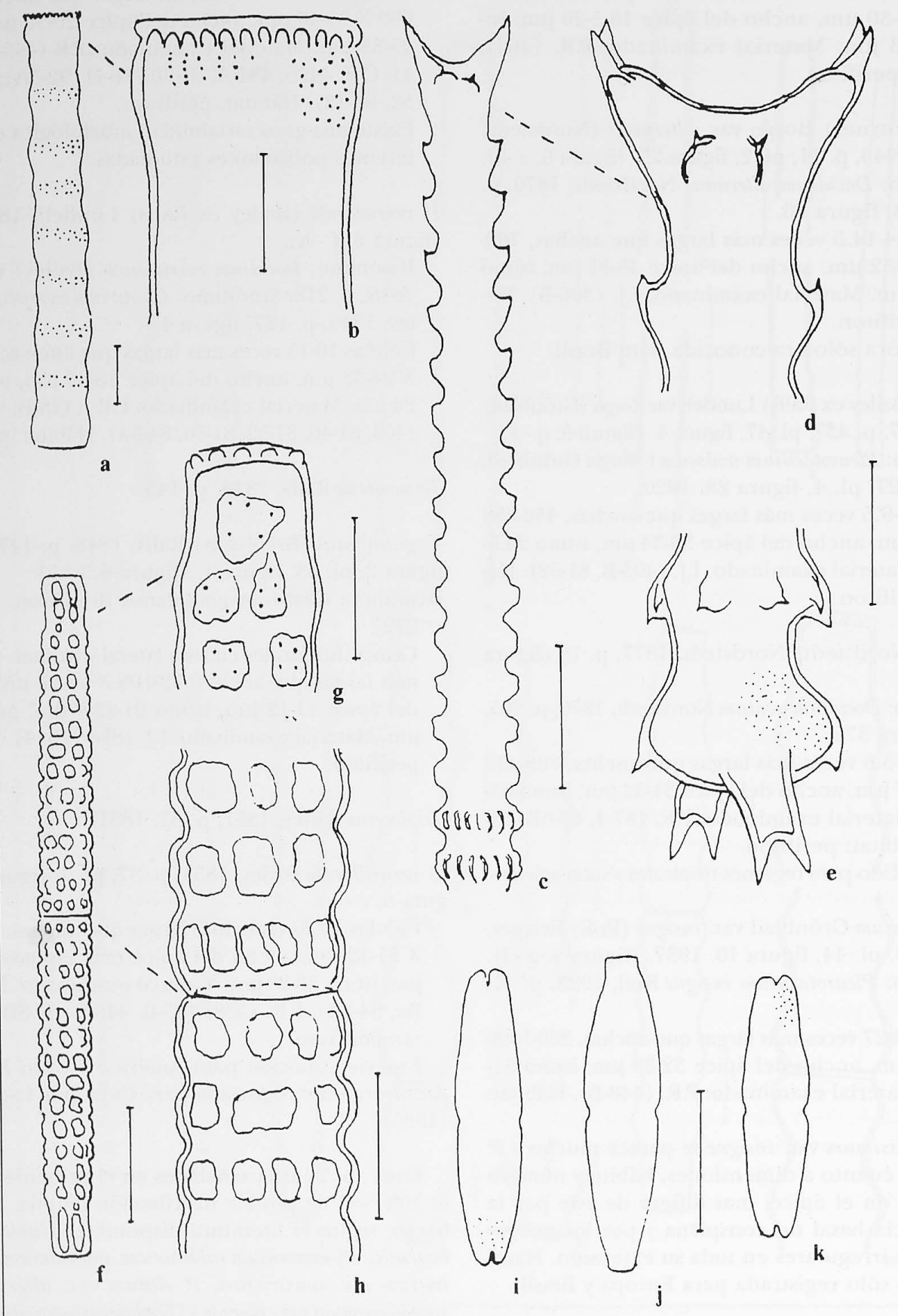

Figura 6. a -b: Pleurotaenium elatum var. alternans; a: hemicélula mostrando poros mucilaginosos; b: detalle del ápice; c - e: Triploceras verticillatum; $\mathrm{d}$ - e: detalle del ápice; $\mathrm{f}$ - h: Pleurotaenium verrucosum; g: detalle del ápice; h: detalle del istmo; i - k: Tetmemorus granulatus; i, k: vista frontal; j: vista lateral. Escalas: d, e, g, h: barra $=20 \mu \mathrm{m}$. b: barra $=25 \mu \mathrm{m}$. Otras: barra $=50 \mu \mathrm{m}$. 
$P$. ehrenbergii var. ehrenbergii y $P$. trabecula presentaron gran variabilidad morfológica.

\section{Taxa registrados para Cuba por otros autores}

Docidium undulatum Bailey, 1851 (Lagerheim,1885, p. 252 sub Docidium dilatatum (Cleve) Lundell). Pleurotaenium caldense Nordstedt, 1877, (Lagerheim, 1887, p. 199 sub $P$. coronulatum var. caldense). $P$. caldense var. cristatum (Turner) Krieger, 1937 (Borge, 1899, p. 15, pl. 1, figura 11 sub $P$. cristatum (Turner) Borge). $P$. elatum (Turner) Borge (Borge, 1899, p. 16, pl.1, figura 13 sub $P$. elatum (Turner) Borge nob. forma) lo considera perteneciente al género Pleurotaenium y no a Docidium (D. elatum Turn.). P. nodulosum (Brébisson ex Ralfs) De Bary,1858, (Lagerheim, 1885, p. 250). P. trabecula (Ehrenberg) ex Nägeli f. metula (Lagerheim) Croasdale, 1975, (Prescott et al. 1975), descrito por Lagerheim (1885, p. 251, pl. 27, figura 30 sub $P$. metula). P. tridentulum (Wolle) West var. borgei Krieger, 1937 (Borge 1899, p. 15, pl. 1, figura 10 sub P.. tridentulum var. capitatum West 1892). Triploceras gracile Bailey, 1851 (Lagerheim ,1885, p. 252 sub P. gracile). T. gracile var. bidentatum (Nordstedt) Krieger, 1937 (Lagerheim, 1885, p. 252 sub P. bidentatum).

\section{Agradecimientos}

A la Dra. Maria Teresa de Paiva Azevedo por las sugerencias brindadas y la lectura crítica del manuscrito. Al Dr. Carlos E. de M. Bicudo por las facilidades en el uso de su biblioteca. A todo el personal de investigación y de apoyo de la sección de Ficología del Instituto de Botánica de São Paulo. A la Red Latinoamericana de Botánica (Chile) por los auxilios y la beca de perfeccionamiento concedida (93-P8) que posibilitó la realización del presente trabajo.

\section{Literatura citada}

Azevedo-Paiva M.T. 1984. O gênero Pleurotaenium (Zygnemaphyceae) no Estado de São Paulo: levantamento sistemático. Tesis Doctoral, Universidade Estadual Paulista, Rio Claro, Brasil, 305 pp.

Bailey J. W. 1846. On some new species of American Desmidieae from the Catskill Mountains. American Journal of Science, and arts, New Haven, N. S. 1:126-127.

Bailey J. W. 1851. Microscopical observations made in South Carolina, Georgia and Florida. Smithsonian Contributions to Knowledge, Washington, 2 (Art.8):1-48.

Bando T. 1988. Haplotaenium, a new genus separated from Pleurotaenium (Desmidiaceae, Chlorophyta). Journal of Japanese Botany 63:169-178.

Barker J. 1869. A new and remarkable species of Penium
(Penium spirostriolatum Bark.). Quarterly Journal of Microscopical Science, London, N.S. 9:194.

Borge O. 1899. Über tropische und subtropische SüsswasserChlorophyceen. Bihang till Kongliga Svenka Vetenskapsakademiens Handlingar 24 (12):1-33.

Borge O. 1903. Die Algen der ersten Regnellschen Expedition, 2: Desmidiaceae. Arkiv för Botanik 1:71-138.

Bourrelly P. y Leboime R. 1946. Notes sur quelques algues d'eau douce de Madagascar. Biologisch Jaarboek: Dodonaea, Antwerpen, 13:75-111, 4 Pls.

Brébisson A. 1839. Sur les préparations nécessaires à l'étude des Algues inférieures, \& Catalogue des espèces connus des Desmidiées, et des Diatomées ou Bacillariées. En: Chevalier. Ed. Des microscopes et de leur usage. Paris , 271-273

Cedergren G. R. 1932. Die Algenflora der Provinz Härjedalen. Arkiv för Botanik, Stockholm, 25A(4): 1-109.

Cleve P.T. 1864. Bidrag till kännedomen om Sveriges sötvattensalger af familjen Desmidieae. Öfuersigt af förhandlingar: Kongliga Svenska Vetenskaps-akademien. Stockholm, 1863, 20 (10): 481-497, Pl. 4.

Couté A. y Tell G. 1981. Ultrastructure de la paroi cellulaire de Desmidiacées au microscope électronique à balayage. Beihefte Nova Hedwigia 68 :1-228.

De Bary A. 1858. Untersuchungen über die Familie der Conjugaten (Zygnemeen und Desmidieeen), $91 \mathrm{pp}$.

Delponte J.B.1876-1878. Specimen Desmidiacerum subalpinarum. Memorie della Reale Accademia delle Scienze di Torino, Turin, Ser. 2, 28(1876):19-208. Pls. 1-5; 30 (1878): 1-186.Pls. 7-23.

Ehrenberg C.G. 1830. Beiträge zur Kenntniss der Organisation der Infusorien und ihrer geographischen Verbreitung besonders in Sibirien. Abhandlungen der Königlichen Akademie der Wissenschaften in Berlin: 1-88.

Ehrenberg C. G. 1838. Die Infusionsthierchen als vollkommene Organismen, Leipzig. 548 pp. 64 Pls.

Grönblad R. 1920. Finnländische Desmideaceen aus Keuru. Acta Societatis pro Fauna et Flora Fennica. Helsinki, 47(4): $1-98$.

Grönblad R. 1924. Observations on some desmids. Acta Societatis pro Fauna et Flora Fennica 55(3):3-18.

Grönblad R. 1945. De algis brasiliensibus, praecipue desmidiaceis in regione inferiore fluminis Amazonas a professore August Ginzberger (Wien) anno MCMXXVII collectis. Acta Societatis Scientiarum Fennicae, nov. Sér. B. Helsingforsiae, 2(6): 1-45.

Grönblad R.; Prowse G.A. y Scott A. M. 1958. Sudanese Desmids. Acta Botanica Fennica, Helsinki, 58:1-82, p.

Kouwets F.A.C. y Coesel P.F.M. 1984. Taxonomic revision of the Conjugatophycean family Peniaceae on the basis of the cell wall ultrastructure. Journal of Phycology 20 (4) :555-562.

Krieger W.1932. Die Desmidiaceen der Deutschen Limnologischen Sunda-Expedition. Archiv für Hydrobiologie. Stuttgart, Supplementband 11:129-230. 
Krieger W' 1937. Die Desmidiaceen Europas mitBerücksichtigung der aussereuropäischen Arten. En Rabenhorst, L. Ed.: Kryptogamen-Flora von Deutschland,Österreich und der Schweiz. Leipzig. AkademischeVerlagesellschaft m.b.H. 13(2), 1.Teil: 1-712 p.

Lagerheim G. 1885. Bidrag till Amerikas Desmidié-flora. Öfversigt af K. Vetenskapsakademiens Förhandlingar 42 (7) $: 225-255$.

Lagerheim G. 1887. Über einige Algen aus Cuba, Jamaica und Puerto Rico. Botaniska Notiser 1887:193-199.

Lundell P.M. 1871. De Desmidiaceis, quae in Suecia inventae sunt, observationes criticae. Nova Acta Regiae Societatis Scientiarum Upsaliensis. Uppsala, Ser. 3, 8:1-100, 5 Tab.

Martínez Almeida V.M. 1989a. Géneros de Desmidiaceae (Chlorophyta) en Isla de la Juventud y Pinar del Río, Cuba. Acta Botánica Cubana 75:1-24.

Martínez Almeida V.M. 1989b. Contribuciones a la revisión del género Micrasterias (Desmidiaceae: Chlorophyta) en Cuba. Acta Botánica Cubana 77:1-14.

Martínez Almeida V.M. 1989c. Desmidiáceas filamentosas (Chlorophyta) de Isla de la Juventud y Pinar del Río, Cuba. Acta Botánica Cubana 84:1-13.

Nägeli C. 1849. Gattungen einzelliger Algen physiologisch und systematisch bearbeitet. Zürich. $8+139$ p.

Nordstedt O. 1870. 18 Fam. Desmidiaceae. En: Warming, E. Ed: Symbolae ad floram Brasiliae centralis cognoscendam, Part. quinta. Videnskabelige Meddelelser fra dansknaturhistorisk Forening i Kjöbenhavn. Copenhagen 1869, III (14/ 15): $95-234$.

Nordstedt O. 1877. Nonnullae algae aquae dulcis brasilienses. Öfversigt af förhandlingar: Kongliga Svenska Vetenskapsakademien. Stockholm, 1877 (3): 15-28.

Prescott G. W.; Croasdale H. T. y Vinyard W. C. 1975.. A Synopsis of North American Desmids. Part II. Desmidiaceae: Placodermae, Section 1.- North American Flora, Ser. 2/ 6, Lincoln. vii +275 pp. Pls. 9-57.
Ralfs J. 1848. The British Desmidiecae. London. xxii +226 p. Roll J. V. 1923. Novye vidy i formy desmidievych vodoroslej, najdennye $v$ Archangel'skoj i Oloneckoj gub. Desmidiaceae novae in gub. Archangelskensi et Olonetzkensi inventae. Botaniceskie Materialy Instituta Sporovy'h Rastenij Glavnogo Botaniceskogo Sada RSFSR. Petrograd. 2:36-46.

Round E.E. 1971. The taxonomy of the Chlorophyta II. British Phycological Journal 6:235- 264

Schaarschmidt G.1883 . Tanulmányok a Magyarhoni Desmidiaceákról.- Magyar Tudományos Akadémia Matematika és Természettudományi Osztályának Kösleményei 18 (1882): 259-280.

West W. 1890. Contribution to the Freshwater Algae of North Wales. Journal of the Royal Microscopical Society, London, 1890, 6:277-306.

West W. 1892. A contribution to the Freshwater Algae of West Ireland. Journal of the Linnean Society: Botany. London, 29:103-216.

West W. y West G.S. 1896. On some North American Desmidiaceae. Transactions of the Linnean Society London Bot.II, 5(5): 229-274.

West W. y West G.S. 1902. A contribution to the freshwater algae of Ceylon. Transactions of the Linnean Society of London, Botany. London, Ser. 2, 6: 123-215, Pls.17-22.

West W'. y West G.S. 1904. A Monograph of the British Desmidiaceae. Vol. I. Ray Society, London, 224 pp. 32 Pls.

Wille N. 1881. Bidrag til Kundskaben om Norges Ferskvandsalger. I. Smaalenenes Chlorophyllophyceer. Christiania Videnskabsselskabs, Förhandlunger 1880:1-72.

Wille N. 1884. Bidrag til Sydamerikas Algflora. I-III.-Bihang til Kongliga Svenska Vetenskaps-akademiens Handlingar: Stockholm 8(18):1- 64.

Wolle F. 1885. Fresh-Water Algae. IX. Bulletin of the Torrey Botanical Club. Pennsylvania, 12(1):1-6.

Wood H. C. 1873. A contribution to the history of the freshwater algae of North America. Smithsonian Contributions to Knowledge, Washington, 19(241):8 + 262 pp. 